\title{
Dynamic Performance Optimization of a 2-DoF Parallel Mechanism based on Comprehensive Acceleration Ellipsoid
}

\author{
Qi Hao ${ }^{1, a^{*}}$, Jing Zhou ${ }^{2, b}$, Jing Xue ${ }^{1, c}$ \\ ${ }^{1}$ Institute of Army Aviation, Beijing 101123, China \\ ${ }^{2}$ Beijing Aerospace Measurement \& Control Technology Co. LTD, Beijing 100041, China \\ ahaoqiv@163.com, 'bj9309@163.com, ‘xuejing1988@126.com
}

Keywords: Parallel mechanism; dynamic performance optimization; comprehensive acceleration ellipsoid; genetic algorithm

\begin{abstract}
In order to optimize the dynamic performance of a 2-DoF planar parallel mechanism used in hybrid machines, comprehensive acceleration ellipsoid (CAE) method is introduced. Considering the inertia, velocity, gravity and external force factors, CAE method can evaluate the dynamic performance more exactly. Based on the dynamic analysis, the optimization based GA method is analyzed. From the simulation, it is proved that the dynamic optimization based on CAE method improves the dynamic performance well.
\end{abstract}

\section{Introduction}

These years, the parallel mechanism with less than 6-DoF has been researched more widely [1][2]. The 2-DoF planar parallel mechanism and 3-DoF spatial parallel mechanism have been considered as the easiest ways to achieve Multi-DoF hybrid machine tools. When being used in a heavy duty high-speed hybrid machine tool, the dynamic performance of the parallel mechanisms must be analyzed and optimized. A number of studies are addressed the analysis of the dynamic performance, such as the generalized inertia ellipsoid (GIE) method [3], the dynamic manipulability ellipsoid (DME) method [4], the acceleration line (AL) method [5], the manipulating force ellipsoid (MFE) method [6], the comprehensive acceleration ellipsoid (CAE) method [7] and so on.

To improve the dynamic performance of a 2-DoF planar parallel mechanism, which is used in a heavy duty hybrid machine tool, the dynamic performance optimization based on CAE is investigated. The CAE method considering the effect of the inertia, speed, gravity and external force factors, can analyze the dynamic performance evaluation of the mechanism more accurately. Based on the CAE method, the dexterous acceleration index (DAI) and comprehensive acceleration dexterity index (CAD), give more comprehensive description of the dynamic performance. The global dexterous acceleration (GDA) and global comprehensive acceleration dexterity (GCAD) can be used in the dynamic performance optimization of the mechanism.

\section{Kinematic analysis and dynamic analysis}

The 2-PRR parallel mechanism consists of a base platform, two legs and a moving platform. By move the active prismatic joints, the moving platform will have two degrees of translational freedom. As shown in Fig. 1, a base coordinate frame which is denoted as $O-X Y$ frame is fixed at the centre of the base platform, a moving coordinate frame $N$-xy is located at the centre of the moving platform.

$q_{i}(i=1,2)$ is the $Y$ coordinate of point $A_{i}$ on the slider, so the movement input vector is

$\boldsymbol{q}=\left[\begin{array}{ll}q_{1} & q_{2}\end{array}\right]^{\mathrm{T}}$

The position vector of point $N$ and $A_{i}$ can be written as

$\boldsymbol{p}=\left[\begin{array}{ll}x & y\end{array}\right]^{\mathrm{T}}$ and $\boldsymbol{A}_{i}=\left[\begin{array}{ll}(-1)^{i} l_{m} & q_{i}\end{array}\right]^{\mathrm{T}}(i=1,2)$

According to the geometrical relationship of the parallel mechanism, there are 


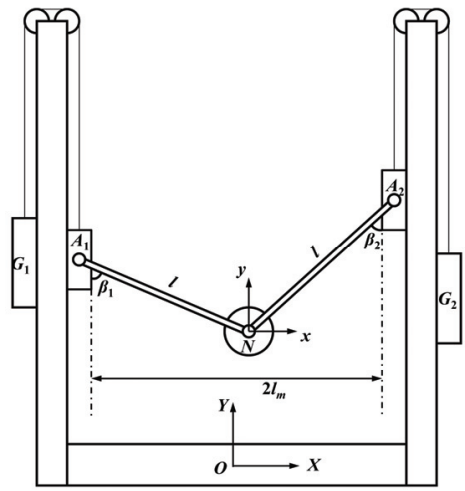

Figure 1. The kinematic model of the 2-PRR parallel mechanism

The inverse kinematics equations of the mechanism can be written as

$q_{1}=y+\sqrt{l^{2}-\left(x+l_{m}\right)^{2}}$ and $q_{2}=y+\sqrt{l^{2}-\left(x-l_{m}\right)^{2}}$

Then taking the derivative of Eq. (3), there are

$\dot{\beta}_{i}=\dot{x} /\left(l \cos \beta_{i}\right)$ and $\dot{q}_{i}=\dot{y}+l \dot{\beta}_{i} \sin \beta_{i}$

From Eq. (5), it can be get that $\dot{q}_{i}=\boldsymbol{J}_{i}\left[\begin{array}{ll}\dot{x} & \dot{y}\end{array}\right]^{\mathrm{T}}$, where $\boldsymbol{J}_{i}=\left[\begin{array}{ll}-\tan \beta_{i} & 1\end{array}\right]$.

Then the relationship between the output and the input velocity vector can be written as

$\dot{\boldsymbol{p}}=\boldsymbol{J} \dot{\boldsymbol{q}}$ where $\boldsymbol{J}=\left[\begin{array}{ll}\boldsymbol{J}_{1}^{\mathrm{T}} & \boldsymbol{J}_{2}^{\mathrm{T}}\end{array}\right]^{\mathrm{T}}$

The velocity vectors of the key points of the parallel mechanism can be written as

$\dot{\boldsymbol{p}}=\left[\begin{array}{ll}\dot{x} & \dot{y}\end{array}\right]^{\mathrm{T}}, \boldsymbol{v}_{A_{i}}=\left[\begin{array}{ll}0 & 1\end{array}\right]^{\mathrm{T}} \dot{q}_{i}$ and $\boldsymbol{v}_{G_{i}}=\left[\begin{array}{ll}0 & -1\end{array}\right]^{\mathrm{T}} \dot{q}_{i}$

Then taking the derivative of Eq. (5), there are

$\ddot{\beta}_{i}=\left(\ddot{x} l \cos ^{2} \beta_{i}+\dot{x}^{2} \sin \beta_{i}\right) /\left(l^{2} \cos ^{3} \beta_{i}\right)$ and $\ddot{q}_{i}=\ddot{y}-\ddot{\beta}_{i} l \sin \beta_{i}-\dot{\beta}_{i}^{2} l \cos \beta_{i}$

Then the acceleration vectors of the key points of the parallel mechanism can be written as

$\ddot{\boldsymbol{p}}=\left[\begin{array}{ll}\ddot{x} & \ddot{y}\end{array}\right]^{\mathrm{T}}, \boldsymbol{a}_{A_{i}}=\left[\begin{array}{ll}0 & 1\end{array}\right]^{\mathrm{T}} \ddot{q}_{i}$ and $\boldsymbol{a}_{G_{i}}=\left[\begin{array}{ll}0 & -1\end{array}\right]^{\mathrm{T}} \ddot{q}_{i}$

The sum of the virtual work done by all forces and torques should be zero. Therefore

$$
\boldsymbol{J}^{-\mathrm{T}} \boldsymbol{\tau}+\sum_{i=1}^{2} \sum_{j=1}^{3}\left[\begin{array}{ll}
\boldsymbol{H}_{i j}^{\mathrm{T}} & \boldsymbol{G}_{i j}^{\mathrm{T}}
\end{array}\right]\left[\begin{array}{l}
\boldsymbol{F}_{i j} \\
M_{i j}
\end{array}\right]+\left[\begin{array}{ll}
\boldsymbol{H}_{N}^{\mathrm{T}} & \boldsymbol{G}_{N}^{\mathrm{T}}
\end{array}\right]\left[\begin{array}{c}
\boldsymbol{F}_{N} \\
M_{N}
\end{array}\right]=\boldsymbol{O}
$$

where, $\boldsymbol{H}_{i j}$ is the partial angular velocity matrix and $\boldsymbol{G}_{i j}$ is the partial velocity matrix of the slider, leg, weight, or moving platform. And where:

$$
\begin{aligned}
& \boldsymbol{F}_{i 1}=m_{\mathrm{S}_{i}}\left(\boldsymbol{a}_{A_{i}}-\boldsymbol{g}\right) \text { and } M_{i 1}=0 \text {; }
\end{aligned}
$$

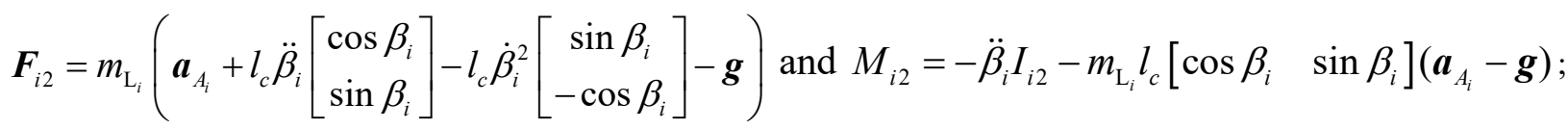

$$
\begin{aligned}
& \boldsymbol{F}_{i 3}=m_{\mathrm{W}_{i}}\left(\boldsymbol{a}_{G_{i}}-\boldsymbol{g}\right) \text { and } M_{i 3}=0 \text {; } \\
& \boldsymbol{F}_{N}=m_{\mathrm{M}}(\ddot{\boldsymbol{p}}-\boldsymbol{g})-\boldsymbol{F}_{e} \text { and } M_{N}=0 \text {. }
\end{aligned}
$$

So the inverse dynamic equation of the parallel mechanism can be written as:

$$
\boldsymbol{\tau}=-\boldsymbol{J}^{\mathrm{T}}\left(\sum_{i=1}^{2} \sum_{j=1}^{3}\left[\begin{array}{ll}
\boldsymbol{H}_{i j}^{\mathrm{T}} & \boldsymbol{G}_{i j}^{\mathrm{T}}
\end{array}\right]\left[\begin{array}{c}
\boldsymbol{F}_{i j} \\
M_{i j}
\end{array}\right]+\left[\begin{array}{ll}
\boldsymbol{H}_{N}^{\mathrm{T}} & \boldsymbol{G}_{N}^{\mathrm{T}}
\end{array}\right]\left[\begin{array}{c}
\boldsymbol{F}_{N} \\
M_{N}
\end{array}\right]\right)
$$

Based on the factors separation, Eq. (11) can also be written as:

$$
\tau=\boldsymbol{M}(\boldsymbol{p}) \ddot{\boldsymbol{p}}+\boldsymbol{C}(\boldsymbol{p}, \dot{\boldsymbol{p}})+\boldsymbol{G}(\boldsymbol{p})+\boldsymbol{F}(\boldsymbol{p})
$$

From Eq. (12), the acceleration performance function (APF) of the parallel mechanism can be get $\ddot{\boldsymbol{p}}=\boldsymbol{M}^{-1} \boldsymbol{\tau}+\ddot{\boldsymbol{p}}_{v}+\ddot{\boldsymbol{p}}_{g}+\ddot{\boldsymbol{p}}_{e}$ 


\section{Dynamic performance optimization based on CAE}

Taking into account the measure of the dynamic performance, the minimum acceleration is an important performance, so the DAI is given based on the CAE method to evaluate the dynamic performance. In order to compare the acceleration performance of different parallel mechanisms, the index of the whole workspace of the mechanism is given too, named GDA. They are defined as

$$
D_{a}=\left\|\boldsymbol{a}_{\min }\right\| \text { and } \eta_{D}=\frac{\int_{W} D_{a} d W}{\int_{W} d W}
$$

A dexterity index named CAD, which is similar to LCI and DCI, is the ratio between the minimum radius and the maximal radius of the $\mathrm{CAE}$ ellipsoid. It can measure the isotropy of the dynamic performance of parallel mechanisms. Similarly, in order to compare the isotropy of the dynamic performance of different parallel mechanisms in the whole workspace, a global evaluation index is proposed based on the CAD, named GCAD. They are defined as follows

$$
\kappa_{a}=\frac{\left\|\boldsymbol{a}_{\min }\right\|}{\left\|\boldsymbol{a}_{\max }\right\|} \text { and } \eta_{D}=\frac{\int_{W} \kappa_{a} d W}{\int_{W} d W}
$$

Genetic algorithm (GA) method can solve the optimization problem of complex multivariable systems. Here the GDA index and the GCAD index are considered together. In order to get the global optimum of dynamic performance in whole workspace, the fitness function is defined as

$$
J=\frac{\mathrm{GDA}}{\mathrm{GDA}_{\text {ori }}}+\frac{\mathrm{GCAD}}{\mathrm{GCAD}_{\text {ori }}}
$$

where $\mathrm{GDA}_{\text {ori }}$ and $\mathrm{GCAD}_{\text {ori }}$ are the original value before the optimization.

In Tab. 1, the geometrical and inertial parameters of the 2-DoF parallel mechanism are given. Then the original distribution of the DAI and CAD get by simulation is shown in Fig. 2 and Fig. 3. And the original value of the GDA and the GCAD are $6.7795 \mathrm{~m} / \mathrm{s}^{2}$ and 0.5213 .

Table 1. The geometrical and inertial parameters of the 2-DoF parallel mechanism

\begin{tabular}{|c|l|c|c|}
\hline Parameter & \multicolumn{1}{|c|}{ Comment } & Original Value & Optimized Value \\
\hline$l$ & The length of the link & $1.350 \mathrm{~m}$ & $1.398 \mathrm{~m}$ \\
\hline$l_{m}$ & The position parameter of the sliders & $1.100 \mathrm{~m}$ & $1.062 \mathrm{~m}$ \\
\hline$m_{\mathrm{S}_{i}}$ & The mass of Slider $i$ & $25.000 \mathrm{Kg}$ & $22.047 \mathrm{Kg}$ \\
\hline$m_{\mathrm{L}_{i}}$ & The mass of Link $i$ & $12.000 \mathrm{Kg}$ & $11.2678 \mathrm{Kg}$ \\
\hline$m_{\mathrm{M}}$ & The mass of the moving platform & $25.000 \mathrm{Kg}$ & $24.311 \mathrm{Kg}$ \\
\hline
\end{tabular}

*The mass of the weight $m_{\mathrm{W}_{i}}$ can be defined by other three masses.

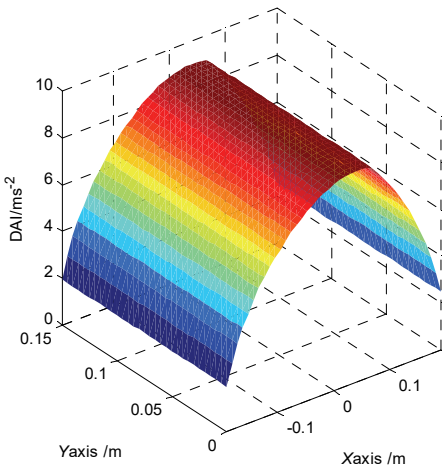

Figure 2. The original distribution of the DAI

After the GA optimization (Fig. 4), the optimized values of the geometrical and inertial parameters of the mechanism are shown in Tab. 1. And the dynamic performance of the parallel mechanism of the mechanism improves in evidence, as shown in Fig. 5 and Fig. 6. The optimized value of the GDA is $8.6097 \mathrm{~m} / \mathrm{s}^{2}$, the optimized value of the GCAD is 0.5759 . 


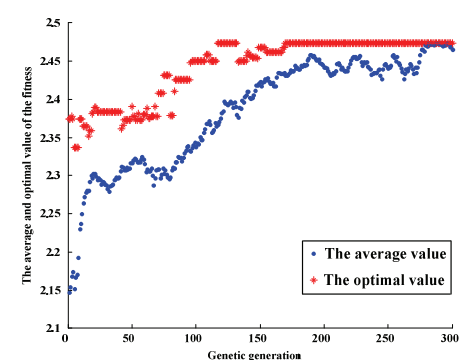

Figure 4. GA optimization based CAE method
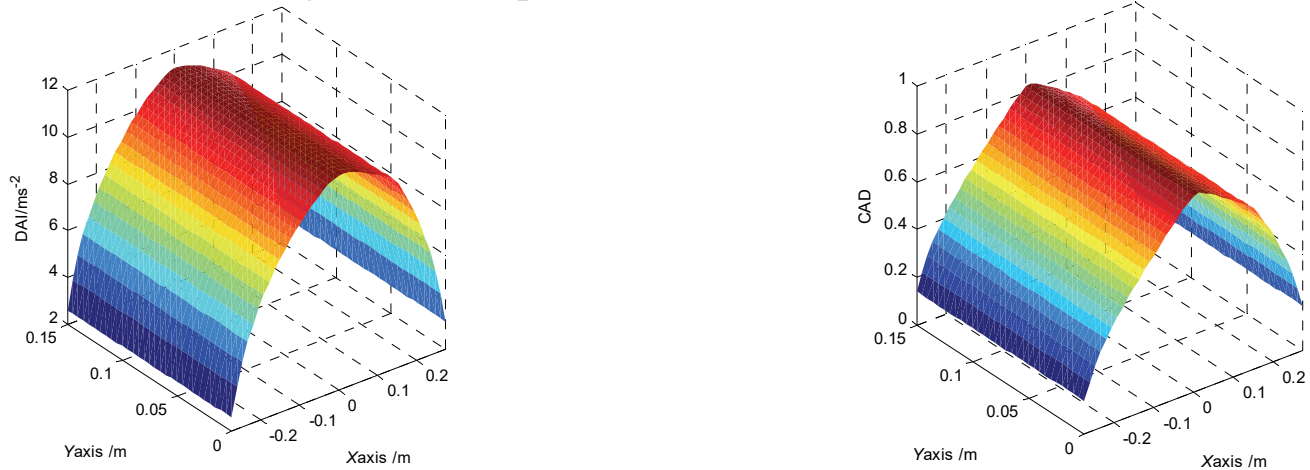

Figure 5. The optimized distribution of the DAI Figure 6. The optimized distribution of the CAD

\section{Conclusions}

By using the virtual work principle method, the inverse dynamic equation and the APF of the 2-DoF parallel manipulator is derived. Furthermore, the dynamic performance optimization based on CAE method is given. The GDA index and the GCAD index are chose as the evaluation indices. From the numerical simulation, it is proved that the GA-based dynamic optimization considering CAE method can improve the dynamic performance of the parallel manipulator well.

\section{Acknowledgment}

This work is supported by the National Nature Science Foundation of China (Grant No. 51405509), all support is gratefully acknowledged.

\section{References}

[1] G. Pritschow. 2000. Parallel kinematic machines (PKM) limitations and new solution. CIRP annals-manufacturing technology, vol. 49(1): 275-280.

[2] Hao Q, Wang L. P, Guan L. W. and Liu Xin-Jun. 2009. Dynamic Analysis of a Novel 3-PSP 3-DoF Parallel Mechanism. Proceedings of the ASME/IFToMM International Conference on Reconfigurable Mechanisms and Robots, London: 309- 314.

[3] Asada H. 1984. Dynamic analysis and design of robot mechanisms using inertia ellipsoids. Proceedings of IEEE international conference on robotics and automation, 1984: 94-102.

[4] Yoshikawa T. 1985. Dynamic manipulability of robot mechanisms. Journal of robotic systems. 2(1): 113-124.

[5] Shiller Z, Sundar S. 1991. Design of robotic mechanisms for optimal dynamic performance. Proceedings of the 1991 IEEE international conference on robotics and automation: 334-339.

[6] Koeppe R, Yoshikawa T. 1997. Dynamic manipulability analysis of compliant motion. Intelligent robots and systems, proceedings of IEEE/RSJ international conference on IROS: 1472-1478.

[7] Hao Q, Guan L. W. and Wang J. X. 2012. Dynamic performance evaluation of a 2-DoF planar parallel mechanism. International journal of advanced robotic systems, Vol. 9, No. 250. 